Revue des patrimoines

\title{
Métiers d'art liés à la restauration et professionnels de la conservation-restauration : deux idéaltypes
}

Restoration master craftsmen and professionals of conservation-restoration, two ideal types

Pierre Leveau

\section{OpenEdition}

Journals

Édition électronique

URL : http://journals.openedition.org/insitu/13611

DOI : 10.4000/insitu.13611

ISSN : 1630-7305

Éditeur

Ministère de la culture

Référence électronique

Pierre Leveau, « Métiers d'art liés à la restauration et professionnels de la conservation-restauration deux idéaltypes », In Situ [En ligne], 30 | 2016, mis en ligne le 22 septembre 2016, consulté le 19 avril 2019. URL : http://journals.openedition.org/insitu/13611 ; DOI : 10.4000/insitu.13611

Ce document a été généré automatiquement le 19 avril 2019

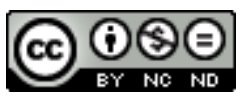

In Situ Revues des patrimoines est mis à disposition selon les termes de la licence Creative Commons Attribution - Pas d'Utilisation Commerciale - Pas de Modification 4.0 International. 


\section{Métiers d'art liés à la restauration et professionnels de la conservation- restauration : deux idéaltypes}

Restoration master craftsmen and professionals of conservation-restoration, two ideal types

Pierre Leveau

\section{Introduction}

1 Le Comité international des musées a reconnu l'existence de la profession de conservateur-restaurateur en 1984 en la distinguant des métiers de l'artisan'. Contrairement à ceux-ci, les conservateurs-restaurateurs ne produisent aucun bien matériel, mais conservent et restaurent les objets hérités du passé. En 1992, ces professionnels ont formé en Europe la Confédération européenne des organisations de conservateurs-restaurateurs (European Confederation of Conservator-Restorers Organisation, Ecco) pour promouvoir leur activité et défendre leurs intérêts, qui coïncident selon eux avec ceux du patrimoine ${ }^{2}$. Mais cette situation s'avère être l'aboutissement d'un long processus de différenciation commencé dans l'Entre-deuxguerres ${ }^{3}$. Dès 1931, les experts de l'office international des musées expliquaient déjà que le meilleur moyen de protéger le patrimoine des méfaits de l'ignorance était d'ouvrir des écoles de restauration et de réglementer l'activité des praticiens, puis de protéger leur titre pour éliminer les autodidactes incompétents ${ }^{4}$. L'institution patrimoniale a partiellement exaucé leurs vœux. Dans le secteur des musées, la professionnalisation de cette activité conduit cependant à une situation que l'on peut juger paradoxale. Faire de la restauration une discipline à part entière a en effet exclu du marché tous les métiers d'art qui n'ont pas séparé cette activité de la confection pour la rattacher à la conservation, comme l'ont fait les conservateurs-restaurateurs. Légalement, les maîtres artisans, qui ont hérité du savoir-faire indispensable à la fabrication de nombreux objets conservés dans les musées, perdent le droit de les restaurer dès qu'ils font partie des 
collections de ces derniers. Comment expliquer ce paradoxe? On répondra en construisant l'idéaltype ${ }^{5}$ de ces activités et en cherchant dans l'histoire les circonstances qui les ont conduits à s'opposer. On rappelle pour cela le rôle qu'ont joué les formations dans ce processus de professionnalisation, en insistant sur la nécessité de replacer la France dans le réseau international de protection du patrimoine pour le comprendre. Qu'est-ce qui distingue finalement les maîtres artisans des métiers d'art et les professionnels de la conservation-restauration? Comment expliquer l'existence d'un clivage aussi marqué entre des praticiens que l'on confond généralement? Pourquoi la France fut-elle l'un des derniers pays à l'opérer?

\section{Distinction d'un métier et d'une profession}

2 Il convient de distinguer les «métiers liés à la restauration du patrimoine » et les "professionnels de la conservation-restauration des biens culturels ». Il s'agit dans le premier cas d'activités dont la liste est définie par l'arrêté du 12 décembre 2003 sur les métiers d'art ${ }^{6}$, tandis qu'il s'agit dans le second d'individus dont les qualifications sont définies par la circulaire $\mathrm{n}^{\circ}$ 2002/021 du 24 décembre 2002 de la loi sur les musées de France $^{7}$. Dans le cas particulier de ces musées, ces dispositions autorisent les maîtres artisans des métiers d'art à effectuer des "restitutions ", mais pas de "restauration ", à l'inverse des professionnels de la conservation-restauration qui ne font pas de restitution, mais restaurent les collections. L'État justifie ces différences en s'appuyant sur le niveau de qualification et le référentiel de formations des praticiens : tandis que les spécialistes des métiers d'art sont titulaires d'un certificat et d'un brevet d'études professionnelles, ou d'un brevet et d'un diplôme des métiers d'art ${ }^{8}$, le diplôme à finalité professionnelle des conservateurs-restaurateurs correspond à un second cycle de l'enseignement supérieur et à un grade de master 9 . Chaque groupe de praticiens a par ailleurs confié à des associations particulières le soin de défendre ses intérêts : il s'agit notamment de l'Union nationale des métiers d'art $^{10}$ pour les métiers liés à la restauration du patrimoine et de la Fédération française des professionnels de la conservation-restauration ${ }^{11}$ pour les praticiens qualifiés par la loi sur les musées. Sur le plan de la pratique enfin, cette distinction se justifie par le fait que les premiers ne séparent pas la restauration de la fabrication et exercent conjointement ces deux activités, tandis que les seconds les ont dissociées et ont lié la première à la conservation pour en faire une discipline à part entière. Les maîtres artisans "sauvegardent » le patrimoine et peuvent voir leur entreprise distinguée par un label de qualité. Les conservateurs-restaurateurs le « conservent » et s'engagent pour cela à suivre un code de déontologie ${ }^{12}$. On voit donc qu'il existe au moins cinq différences spécifiques entre les «métiers liés à la restauration» et les "professionnels de la conservation-restauration ». Mais la frontière qui sépare les deux catégories est naturellement poreuse. Un «artisan d'art» peut devenir un «professionnel» de la conservation-restauration, par validation des acquis de l'expérience ou agrément de la commission d'habilitation prévue par la loi sur les musées de France. Inversement, de nombreux professionnels de la conservation-restauration cotisent au régime social des artisans plutôt qu'à celui des professions libérales et restent attachés à la tradition des métiers d'art. Ces traits distinctifs définissent donc moins des «catégories » que des ensembles flous ou des idéaltypes. Le fait qu'un praticien en soit porteur ne suffit pas à le situer d'un côté ou de l'autre de la frontière qui sépare les deux groupes. 
3 Les métiers d'art et la conservation-restauration diffèrent aussi par leur histoire. Celle des métiers est naturellement plus longue. C'est une "tradition » au sens où elle a pour vocation de transmettre des savoir-faire acquis en atelier liés à la production d'objets manufacturés. On peut l'enraciner dans l'histoire nationale par un mécanisme de filiation inversé bien connu des sociologues ${ }^{13}$. Elle se confond depuis 1965 avec celle de la Société d'encouragement des métiers d'art ${ }^{14}$ qui succéda à la Société d'encouragement à l'art et l'industrie fondée en $1889^{15}$. Mais on peut aussi la rattacher à trois autres traditions bien plus anciennes: celle des manufactures royales créées au XVII ${ }^{\mathrm{e}}$ siècle par Colbert dans différents secteurs du mobilier et de la métallurgie, celle du compagnonnage, récemment inscrit par l'Unesco sur la liste représentative du patrimoine culturel immatériel de l'humanité ${ }^{16}$ et celle enfin des confréries, des guildes et des corporations qui structuraient la société de l'Ancien Régime depuis la fin du Moyen Âge ${ }^{17}$. Celle à laquelle se rattachent les professionnels de la conservation-restauration française est en revanche bien différente ; le texte fondateur de leur profession n'est pas d'inspiration nationale, mais internationale. En 1984, le Comité pour la conservation du Comité international des musées a en effet choisi de définir cette profession pour rompre avec les métiers d'art ${ }^{18}$. À la différence de ceux-ci, les conservateurs-restaurateurs ne produisent pas d'objets, mais conservent ceux qui ont été patrimonialisés en raison de leur valeur historique, artistique ou scientifique. Les organisations internationales de protection des biens culturels leur ont donné ce rôle après la Seconde Guerre mondiale ${ }^{19}$, à la suite de celles qui les ont précédées à l'époque de la Société des nations et de l'invention du patrimoine commun de l'humanité ${ }^{20}$. On voit donc qu'il s'agit de deux lignées radicalement différentes: tandis que l'une est ancrée dans notre histoire depuis la fin du Moyen Âge, l'autre a été instituée par la communauté internationale au milieu du $\mathrm{xx}^{\mathrm{e}}$ siècle.

4 Ajoutons à cela que les métiers liés à la restauration forment une tradition vivante, tandis que les professionnels de la conservation-restauration se rattachent à un paradigme scientifique. Ils s'opposent diamétralement de ce point de vue. Alors qu'une tradition vivante vise à transmettre des pratiques héritées du passé, sans rompre avec la création pour l'enrichir de nouveaux procédés, un paradigme scientifique a pour fonction de produire des connaissances en adoptant une démarche rationnelle et de critiquer toutes celles qui ont porté sur son objet par le passé. Leur confrontation dans le domaine de la restauration a conduit les conservateurs-restaurateurs à refuser l'héritage théorique de leurs prédécesseurs et à cliver les deux groupes. L'ethos des praticiens diffère aussi sur ce point. L'État encourage par loi du 2 août 2005 les artisans à transformer leur savoir-faire en un capital immatériel pour obtenir le label de " patrimoine vivant ${ }^{21}$ ". Mais il impose à l'inverse par contrat aux conservateurs-restaurateurs qui interviennent sur les collections nationales la cession de leurs droits sur la documentation et les actifs immatériels produits dans ce cadre commercial. Les pouvoirs publics ne cultivent pas le même ethos des deux côtés de la frontière qui sépare les deux camps: la propriété intellectuelle et la capitalisation pour les uns, la recherche désintéressée et le souci du vrai pour les autres. Le concept de « restauration » change aussi de sens lorsqu'on passe d'un camp à l'autre ${ }^{22}$ : tandis que du côté des métiers d'art " restaurer » signifie remettre un objet dans son état initial en utilisant des matériaux semblables à ceux de l'original, conformément au sens usuel du terme, cela veut dire pour les conservateursrestaurateurs améliorer la compréhension de l'objet, c'est-à-dire rétablir son fonctionnement sémiotique en utilisant pour cela des matériaux aux propriétés physicochimiques spécifiques distinctes de ceux de l'original ${ }^{23}$. On voit donc que ces praticiens ne 
donnent pas le même sens à ce terme. C'est pourquoi les défenseurs des métiers d'art et ceux de la conservation-restauration ont parfois du mal à se comprendre : leurs objectifs, leur terminologie et leurs méthodes sont radicalement différents. Ils n'ont ni le même jeu de langage ni la même conception du patrimoine. En dépit de leurs airs de famille, ils n'ont rien à voir et n'ont pas la même histoire.

Figure 1

\begin{tabular}{|c|c|c|c|c|}
\hline \multicolumn{5}{|c|}{ DISTINCTION } \\
\hline $\mathbf{N}^{\circ}$ & TRAITS & $\begin{array}{l}\text { Métier d'art lié à la } \\
\text { restauration }\end{array}$ & $\begin{array}{c}\text { Professionnel de la } \\
\text { conservation-restauration }\end{array}$ & \multirow{6}{*}{ 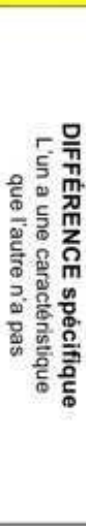 } \\
\hline 1 & Réglementation & $\begin{array}{l}\text { Liste fixée par l'arrêté du } 12 \\
\text { décembre } 2003 \\
\text { (ECOA03200225A) }\end{array}$ & $\begin{array}{l}\text { Critères définis par la } \\
\text { circulaire n²002/021 du } 24 \\
\text { décembre } 2002\end{array}$ & \\
\hline 2 & Champ & $\begin{array}{l}\text { Autorisés à effectuer des } \\
\text { restitutions pour les Musées } \\
\text { de France }\end{array}$ & $\begin{array}{c}\text { Autorisés à restaurer les } \\
\text { biens des collections des } \\
\text { Musées de France }\end{array}$ & \\
\hline 3 & Qualification & $\begin{array}{l}\text { Certificat ou brevet d'études } \\
\text { professionnelles (IV): } \\
\text { brevet ou diplôme des } \\
\text { métiers d'art (III) }\end{array}$ & $\begin{array}{l}\text { Diplóme à finalité } \\
\text { professionnelle } \\
\text { correspondant à la fin du } \\
\text { second cycle universitaire (I) }\end{array}$ & \\
\hline 4 & Critère & $\begin{array}{l}\text { Label de qualité pour les } \\
\text { entreprises (Epv, Qualibat) }\end{array}$ & $\begin{array}{l}\text { Code de déontologie } \\
\text { professionnel (Ecco, Icom) }\end{array}$ & \\
\hline 5 & Organisation & $\begin{array}{c}\text { Union nationale des métiers } \\
\text { d'art (Unma): } \\
\text { Confédération française des } \\
\text { métiers d'Art (Cfma); Etc }\end{array}$ & $\begin{array}{l}\text { Fédération française des } \\
\text { professionnels de la } \\
\text { conservation-restauration } \\
\text { (Ffc- } r) \text {; Araafu ; Etc }\end{array}$ & \\
\hline 6 & Réseau & $\begin{array}{c}\text { National avant d'étre } \\
\text { international (Manufactures) }\end{array}$ & $\begin{array}{c}\text { International avant d'être } \\
\text { national (Iccrom) }\end{array}$ & \multirow{5}{*}{ 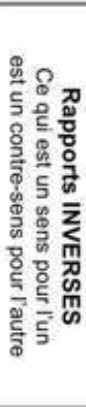 } \\
\hline 7 & Histoire & $\begin{array}{l}\text { Attachement à une tradition } \\
\text { séculaire et vivante }\end{array}$ & $\begin{array}{c}\text { Rattachement à un } \\
\text { paradigme scientifique }\end{array}$ & \\
\hline 8 & Activité & $\begin{array}{l}\text { Associe la fabrication et la } \\
\text { restauration et produisent } \\
\text { des biens matériels } \\
\text { (artisanat) }\end{array}$ & $\begin{array}{l}\text { Dissocie la fabrication et la } \\
\text { restauration et prodiguent } \\
\text { des conseils dans lintérêt } \\
\text { public (libéral) }\end{array}$ & \\
\hline 9 & Pratique & $\begin{array}{c}\text { Restaurer signifie remettre } \\
\text { l'objet dans l'état le plus } \\
\text { proche de l'original. }\end{array}$ & $\begin{array}{c}\text { Restaurer signifie rendre } \\
\text { compréhensible la } \\
\text { signification de l'objet. }\end{array}$ & \\
\hline 10 & Éthos & $\begin{array}{l}\text { Acquerir un droit patrimonial } \\
\text { et conserver un secret de } \\
\text { fabrication. }\end{array}$ & $\begin{array}{l}\text { Céder son droit patrimonial et } \\
\text { une partie de sa } \\
\text { documentation. }\end{array}$ & \\
\hline
\end{tabular}

Distinction entre « profession » et « métier » de la restauration.

(c) Pierre Leveau.

\section{De la réfaction à la restauration}

Il suffit de s'intéresser à l'histoire de l'enseignement de la restauration des œuvres d'art pour s'en convaincre. Rappelons sommairement que le paradigme de la conservationrestauration demande de n'effectuer aucune intervention sans examen scientifique et de les documenter systématiquement en constituant des équipes pluridisciplinaires, de privilégier la conservation et de minimiser la restauration en utilisant des matériaux différents des originau $\mathrm{x}^{24}$. Il fut officiellement adopté par la communauté internationale le 10 octobre 1932, lorsque la Société des nations recommanda à ses membres d'appliquer les résolutions que des experts de l'office international des musées avaient prises à l'issue des conférences de Rome et d'Athènes sur la conservation et la restauration du patrimoine ${ }^{25}$. La France y avait activement participé et poursuivait à l'époque la modernisation de ses institutions. En 1931, elle avait doté le musée du Louvre d'un laboratoire de recherche scientifique et avait réformé en 1935 sa commission de restauration ainsi que le fonctionnement de ses ateliers ${ }^{26}$. C'est dans ce contexte qu'en 1936 le directeur général des Beaux-Arts demanda au chef de l'atelier de restauration des 
peintures du musée du Louvre, J.-G. Goulinat, de soumettre au ministre un projet de création d'école de restauration de peinture. Sa création devait achever la modernisation de l'institution muséale et répondre aux critiques du public sur son opacité ${ }^{27}$. Ce projet s'inscrivait à la suite d'une réflexion plus vaste, orchestrée par l'office international des musées qui avait commandé quatre ans plus tôt une enquête sur la formation professionnelle des restaurateurs ${ }^{28}$. J.-G. Goulinat projetait dans ce cadre de moderniser l'activité sans rompre avec le passé. Il proposait d'ouvrir l'école aux seuls diplômés des Beaux-Arts et de compléter leur formation initiale par un enseignement de deux ans à la fois théorique et pratique, qui les initierait à l'examen scientifique des œuvres et aux nouvelles méthodes préconisées par l'office international des musées. L'objectif n'était donc pas de rompre avec la tradition des peintres-restaurateurs, mais de la prolonger en mettant à jour le référentiel de compétence des praticiens pour les adapter à leur nouvel environnement.

Mais le projet de J.-G. Goulinat fut ajourné en 1937 pour des raisons budgétaires, comme celui de J.-B. Le Brun en 1802. La direction des Beaux-Arts ne put tirer profit de la réforme de l'enseignement technique engagée à l'époque par le ministère de l'Éducation nationale ${ }^{29}$. Elle continua donc à faire fonctionner le nouveau paradigme de la conservationrestauration sur l'ancien régime des corporations de métiers contrôlées par l'État, tout en refusant leur offre de cogestion pour conserver la maîtrise d'œuvre des travaux de restauration. En 1935, elle choisit de maintenir au Louvre l'ancien système de recrutement des restaurateurs par concours institué au siècle passé. L'inscription des lauréats sur une liste d'aptitude donnait aux conservateurs la possibilité de choisir parmi eux les praticiens auxquels ils confieraient les œuvres ${ }^{30}$. En 1936, le directeur des Musées nationaux écarta ainsi l'offre du Syndicat des peintres-restaurateurs qui proposait d'organiser pour eux ce concours de recrutement ${ }^{31}$. En l'absence d'école et de diplôme certifiant la compétence des membres du syndicat, les Musées nationaux n'avaient aucune raison de leur transférer cette prérogative qui aurait transformé leur organisation en conseil de l'ordre de la profession. En 1941, le conservateur-adjoint des peintures du Louvre, G. Bazin, imagina résoudre cette difficulté ${ }^{32}$. Le régime de Vichy venait de réhabiliter les corporations supprimées sous la Première République ${ }^{33}$. Le nouveau projet de création d'école de restauration que G. Bazin soumit à sa direction reprenait dans cette perspective celui de J.-G. Goulinat et ajoutait que l'État pourrait s'appuyer sur les professionnels qu'elle aurait diplômés pour instituer un conseil de l'ordre de la profession et contrôler ainsi ce secteur d'activité, comme c'était déjà le cas pour l'architecture et la médecine. Mais ce projet fut également oublié pour des raisons politiques. Il fallut finalement attendre la création du ministère chargé des Affaires culturelles en 1959 pour qu'un corps de « restaurateur spécialisé » soit officiellement créé dans la fonction publique, par application d'un décret de 1961 sur le reclassement des ouvriers professionnels qui prévoyait la définition de spécialités pour chaque catégorie ${ }^{34}$.

7 En 1965, ce progrès social plaça paradoxalement la France dans une position difficile parce qu'il témoignait aussi du retard qu'elle accusait après-guerre dans le champ de la conservation du patrimoine ${ }^{35}$. Entre 1964 et 1966, tous les établissements qui dépendaient $\mathrm{du}$ ministère de la Culture où existaient des ateliers de restauration recrutèrent sur concours le personnel de ces corps et firent ainsi de la restauration une spécialité des métiers d'art ${ }^{36}$. Dès 1964, l'administration générale du Mobilier national, des manufactures des Gobelins, de Beauvais et de la Savonnerie appliqua ce décret, suivie par les Archives de France et les Musées de France en 1965, la direction des Bibliothèques 
enfin en $1966^{37}$. Le concours était ouvert aux titulaires d'un brevet ou d'un certificat d'études professionnel et aux ouvriers qu'employaient déjà ces établissements. Celui qu'organisèrent les Musées de France proposait différentes spécialités - arts du bois, du métal, de la céramique, du textile et d'autres matériaux ${ }^{38}$ - à l'exclusion de la peinture, pour laquelle le concours institué en 1935 était maintenu. Mais tandis que la restauration devenait en France une spécialité des métiers d'art, elle s'en détachait au même moment sur le plan international. Dès 1950, le Comité international des musées qui avait succédé à l'office international avait rappelé son attachement au paradigme de la conservationrestauration constitué dans l'Entre-deux-guerres. Comme leurs prédécesseurs, ses membres résolurent de défendre la conservation contre la restauration ${ }^{39}$, quinze ans avant que ce principe soit à son tour inscrit dans la charte de Venise adoptée en 1965 par le Conseil international des monuments et des sites ${ }^{40}$. Soucieux de se donner les moyens de leurs fins, ces organisations constituaient simultanément des groupes de travail sur les formations pour enseigner aux praticiens de nouvelles méthodes conformes à leurs principes. Cette mobilisation qui intéressait tous les secteurs du patrimoine était à l'époque orchestrée par le Centre international pour l'étude et la préservation des biens culturels (Iccrom), créé à Rome en 1959 sous l'égide de l'Unesco ${ }^{41}$, dont l'une des missions était la formation. En 1961, ses premiers travaux conduisirent d'abord l'Institut international de conservation à consacrer une section entière de son congrès à cette question $^{42}$, puis le Comité international des musées à l'imiter lors de son assemblée triennale en $1965^{43}$. C'est ce qui mit la France dans une situation paradoxale. Tandis qu'elle faisait de la restauration du patrimoine une «spécialité » des métiers d'art, la communauté internationale prenait la même année la décision de les séparer pour faire de cette activité une « discipline » à part entière. Il lui fallait poursuivre la réforme de ses institutions pour rejoindre la communauté patrimoniale qui avait progressé sans elle après-guerre.

\section{De la restauration à la conservation}

Il fallut finalement attendre la loi Faure et la réforme de l'Université ${ }^{44}$ en 1968 pour que le réseau des "métiers d'art » et celui de la "conservation-restauration ", qui étaient déjà séparés sur le plan des activités, le soient aussi sur celui de la formation. Deux événements indépendants expliquent que le projet de créer une école de restauration ait pu aboutir à cette époque. Le premier est la série de réformes engagée par le ministère de l'Éducation nationale pour satisfaire les revendications des étudiants sur la formation professionnelle à l'Université. Le second est l'élaboration du VI ${ }^{e}$ Plan de développement économique et social de la France pour lequel le ministère en charge réunit un groupe de travail sur les musées qui fit état des besoins de l'institution muséale dans le domaine de la formation des restaurateurs ${ }^{45}$. Le groupe s'appuyait sur les travaux menés à l'Iccrom depuis sa création et sur un rapport que le ministre de la Culture avait commandé à $\mathrm{G}$. Bazin sur la question ${ }^{46}$. Le $\mathrm{VI}^{\text {e }}$ Plan tint compte de leurs demandes et fut approuvé en 1971. Il prévoyait pour les satisfaire la création d'un Institut de restauration, dont l'activité et les missions restaient à préciser ${ }^{47}$. C'est sa création qui aboutit dix ans plus tard au clivage définitif du réseau des métiers d'art et de ceux de la conservationrestauration.

Mais ce clivage ne se fit pas de la même façon dans tous les secteurs ni dans toutes les écoles. La première formation ouvrit en 1973, à l'UER (unité d'enseignement et de 
recherche) d'histoire de l'art et d'archéologie de l'université de Paris 1 , après que le ministère de l'Éducation nationale avait institué en 1971 le diplôme de maîtrise des sciences et techniques (MST) qui offrait aux étudiants la formation professionnalisante réclamée lors des grèves ${ }^{48}$. La MST de Paris 1 devait former les professionnels de la conservation-restauration dont les compétences répondissent aux besoins de différentes institutions susceptibles de leur offrir un débouché, qu'il s'agisse du CNRS ou d'autres organismes dépendants de l'Unesco et du ministère de la Culture ${ }^{49}$. La formation durait quatre ans à l'époque et correspondait à la fin du second cycle d'études universitaires. La MST de deux ans était accessible aux titulaires d'un Deug à mention spécifique; elle comprenait un tiers d'enseignements technologiques et scientifiques en plus des cours théoriques et de trois mois de stage. Mais le prérequis du baccalauréat imposé par l'Université interdisait aux diplômés des métiers d'art d'y avoir accès. Il cliva ainsi leurs réseaux et ceux de la conservation-restauration qui se développaient à l'époque. La création en 1977 de l'Institut français de restauration des œuvres d'art (Ifroa) contribua cependant à résorber ce clivage ${ }^{50}$. Indépendant de l'Université, l'établissement installé au Mobilier national et voulu par le président Giscard d'Estaing fut une association de loi de 1901 avant d'être rattaché au ministère de la Culture. Il n'imposait de ce fait aucun prérequis à ses étudiants, recrutés sur concours quel que soit leur niveau d'études. Loin de l'Unesco et du CNRS, ses responsables travaillaient en étroite collaboration avec différentes directions du patrimoine qui voulaient former les spécialistes de la restauration dont la France avait besoin. Sans prérequis ni visée universelle, l'Ifroa était ouvert aux artisans des métiers d'art et proposait une formation continue d'un an qui leur permettait d'acquérir les compétences justifiant leur rattachement aux réseaux de conservation-restauration dont le développement s'accélérait ${ }^{51}$. La formation initiale qu'il proposait durait quatre ans, comme à la MST de Paris 1. Mais les étudiants y choisissaient immédiatement une spécialité, ce qui distingue encore aujourd'hui les deux formations.

En dépit des apparences, l'Ifroa fut cependant l'artisan de la rupture avec les métiers d'art tout autant que l'Université. Sa création fut en effet le dernier épisode d'un romanfeuilleton commencé avec le VI $\mathrm{V}^{\mathrm{e}}$ Plan de développement, au cours duquel différents acteurs se disputaient la réalisation du projet que le groupe-Musée y avait inscrit. Tandis que la MST de Paris 1 ouvrait ses portes, le ministère de la Culture commanda à Édith Cresson un rapport sur l'administration du futur Institut dont le Plan avait approuvé la création $^{52}$. Mais le document qu'elle remit en 1974 à la direction des Musées nationaux fut vite oublié. Le tableau qu'il dressait des services français de conservation, après avoir procédé à l'audit de ses services pour les comparer avec ceux d'autres pays, était en effet catastrophique. Les conservateurs français ne pouvaient selon elle diriger l'Institut de restauration prévu par le Plan, car leur formation était surtout historique alors que sa direction demandait des compétences scientifiques et techniques. Son rapport plaçait malheureusement le projet dans l'impasse, mais la création en 1965 de la Société d'encouragement aux métiers d'art (Sema) qui succéda à la Seai, le relança deux ans plus tard. En 1976, Valéry Giscard d'Estaing commanda en effet au nouveau président de la Sema, P. Dehaye, un rapport sur les difficultés des métiers d'art ${ }^{53}$. L'industrie qui avait favorisé l'enseignement des arts appliqués au XIX ${ }^{e}$ siècle se retournait contre l'artisanat au $\mathrm{Xx}^{\mathrm{e}}$, si bien qu'il devenait urgent de prendre une série de mesures pour éviter la perte des savoir-faire qui faisaient rayonner la France à l'étranger depuis Colbert. Considérant que la restauration ne se séparait pas de la fabrication dans la tradition des métiers d'art, P. Dehaye proposa de relancer dans ce cadre le projet de création de l'Institut inscrit au 
$\mathrm{VI}^{e}$ Plan, mais en révisant ses missions et en s'appuyant sur le chiffrage du rapport d'É. Cresson.

11 Dans ce contexte, l'établissement ne devait pas seulement ancrer le paradigme de la conservation-restauration en France en formant de nouveaux professionnels, mais aussi sauvegarder certains savoir-faire indispensables à l'entretien du patrimoine, impossibles à professionnaliser parce que n'offrant pas assez de débouchés. L'Institut devait, selon P. Dehaye, avoir pour mission de concilier la tradition vivante des métiers d'art et le paradigme de la conservation-restauration pour préserver toutes les composantes $d u$ patrimoine français. C'est ainsi que la création de la Sema permit de relancer un projet qui avait été inspiré par l'Iccrom, dont le paradigme rompait avec ses pratiques ${ }^{54}$. Il aboutit en 1977, lorsque fut enfin réglée la question du recrutement des enseignants consistant à savoir comment rompre avec les anciennes pratiques pour en instaurer de nouvelles, sachant que cette rupture ne pouvait être totale puisqu'il s'agissait aussi de sauvegarder les métiers d'art. La partie appliquée de l'enseignement aurait pu être confiée aux restaurateurs spécialistes, dont les corps venaient d'être créés par le ministère de la Culture. Mais le directeur du Mobilier national, J. Coural, écarta cette option en 1976 lors de son audition par la Commission interministérielle pour la conservation du patrimoine ${ }^{55}$. Considérant que les restaurateurs spécialistes des métiers d'art confondaient restauration et réfaction, alors qu'il s'agissait de deux opérations différentes, il en concluait qu'on ne pouvait leur confier l'enseignement d'une discipline qu'ils ignoraient en réalité. Si la conservation du patrimoine passait par la sauvegarde des métiers d'art, elle imposait aussi de séparer la fabrication et la restauration pour faire de cette dernière une discipline à part entière. L'état alarmant d'une partie du patrimoine national acheva de persuader la Commission du bien-fondé de l'argument et permit aux conservateurs acquis au paradigme de l'Iccrom de piloter le projet qui leur avait échappé un instant ${ }^{56}$. C'est ainsi que l'Ifroa fut finalement créé avec le concours de la Sema, mais en rompant avec elle ${ }^{57}$.

12 En 1980 enfin, tandis que débutait l'Année du Patrimoine, la France était donc en mesure de suivre les recommandations du paradigme qu'elle avait contribué à définir dans l'Entre-deux-guerres. Mais elle s'en écartait encore sur un point, pourtant capital : faute d'école et de centre de restauration en province, elle ne pouvait protéger le patrimoine national de la même façon sur tout le territoire. L'Ifroa combla cette lacune dans la décennie qui suivit, mais à l'insu de son plein gré. En 1981, le directeur de l'école municipale des Beaux-Arts d'Avignon, G. Casazza, choisit en effet d'ouvrir un département de restauration de peinture de chevalet pour répondre à la demande du ministère de la Culture qui avait créé en 1978 un diplôme national supérieur d'expression plastique $^{58}$ et encourageait à l'époque les écoles d'art à se doter de parcours à mentions spécifiques ${ }^{59}$. L'intérêt que suscita à Avignon l'exposition pédagogique organisée par le musée du Petit Palais autour de la restauration des primitifs italiens de la collection Campana ${ }^{60}$ décida la municipalité à cautionner son projet, qui obtint par ailleurs le soutien de l'Ifroa pour d'autres raisons. Après l'arrivée de la gauche au pouvoir en 1981, l'établissement voulu par Valéry Giscard d'Estaing fut en effet rattaché à la délégation aux Arts plastiques et dut renforcer ses positions encore très fragiles. Sachant que la conservation des œuvres dans les musées de province était depuis la Révolution un argument en faveur de l'ouverture des écoles de restauration ${ }^{61}, \mathrm{~J}$. Coural choisit d'étendre hors de Paris le réseau de l'Institut et d'en faire ainsi un acteur incontournable de la décentralisation culturelle voulue par François Mitterrand et Jack Lang ${ }^{62}$. Ce 
positionnement stratégique permit à la Ville d'Avignon de signer en 1983 une convention avec la délégation aux Arts plastiques, liant l'école des Beaux-Arts à l'Ifroa. L'Institut s'engageait à assister scientifiquement et matériellement l'école dont le diplôme de «technicien supérieur de restauration » serait en retour reconnu par le ministère de la Culture. La convention devait éviter l'éclosion de formations dangereuses en province susceptibles de perpétuer les pratiques des métiers d'art avec lesquelles le directoire de l'Ifroa voulait rompre. Elle autorisait pour cela l'Institut à définir le programme du département de restauration des écoles, à recruter ses enseignants et à évaluer ses étudiants ${ }^{63}$. La formation devait durer trois ans et être complétée par une dernière année de stage à l'Institut, donnant aux étudiants des écoles d'art de province le même niveau que ceux de l'Institut ou de la MST.

Le fait que la convention ait été signée en 1983 ne doit rien au hasard. Le directeur de l'école régionale des Beaux-Arts de Tours, 0 . Seguin, s'était en effet rapproché de la même façon de l'Ifroa en 1982 et avait obtenu l'ouverture d'un département restauration dans son établissement, mais en sculpture - sa spécialité. Comme son collègue d'Avignon, il voulait ainsi répondre aux nouvelles directives de l'Inspection générale des enseignements artistiques. Les deux écoles signèrent la même convention mais ne proposèrent cependant pas les mêmes formations. L'Ifroa offrait à toutes deux une assistance scientifique et technique pour une durée de trois ans et s'engageait à reconnaître leur diplôme en échange de ce contrôle. Les postes d'enseignants y furent attribués sur concours à d'anciens élèves de l'Institut dépêchés à sa demande pour encadrer la formation en rompant avec les anciennes pratiques des métiers d'art. Cette tutelle explique la remarquable cohérence du projet scientifique de toutes les filières de conservation-restauration créées à l'époque: ce fut l'œuvre d'un même ingénieur centralien, G. Delcroix, qui assura d'abord la direction scientifique de la MST de Paris 1, puis celle de l'Ifroa et des départements d'Avignon et Tours. Ces deux écoles firent cependant des choix différents. Tandis que les étudiants d'Avignon suivaient les trois années du cursus de conservation-restauration après une année de propédeutique, ceux de Tours se spécialisaient immédiatement comme à l'Ifroa où ils étaient supposés achever leur formation.

\section{De la conservation à la prévention}

Mais au-delà des équipes pédagogiques et de leur administration, ces formations se distinguaient surtout par les stages et les débouchés qu'elles offraient. À Paris, ce point crucial prit immédiatement la forme d'une rivalité. Dès la création de l'Ifroa en 1977, le nouveau responsable de la MST, J.-P. Sodini, proposa à J. Coural de conserver à l'université la filière de conservation-restauration des objets archéologiques - l'UER d'histoire de l'art et d'archéologie de Paris 1 étant particulièrement compétente dans ce secteur - et de réorienter les étudiants des autres filières vers l'Institut. Le refus de l'Ifroa déclencha une guerre de dix ans entre les deux formations, qui s'explique non seulement par la psychologie de ses acteurs, mais aussi par le désir des Musées nationaux de ne laisser entrer sur le marché du travail que les restaurateurs qu'ils avaient contribué à former. Il fallait non seulement écarter les praticiens sans théorie, ne distinguant pas la réparation et la restauration, mais aussi les théoriciens sans pratique, dont le savoir était abstraitement séparé des œuvres. Trois séries d'événements mirent par la suite fin à cette guerre et stabilisèrent le réseau de formation qui émergeait. En 1988, l'Inspection 
générale des musées classés et contrôlés choisit d'abord d'apporter son soutien à l'université puis aux écoles d'Avignon et Tours. La décentralisation culturelle renforçait à cette époque ses positions et celles de son service de restauration qui développait en province un réseau d'ateliers décentralisé sur un modèle radicalement différent de celui que proposait le service de restauration des Musées nationaux installé au Louvre. Les quatre écoles avaient une importance capitale dans ce dispositif, puisque ce réseau d'ateliers ne pouvait fonctionner que s'il existait suffisamment de praticiens diplômés pour y travailler. C'est le choix de ce modèle conçu par R. Guilly qui acheva d'ancrer le paradigme de la conservation-restauration en France ${ }^{64}$. En 1987, la convention qui liait la délégation aux Arts plastiques aux Villes d'Avignon et Tours arrivait à échéance et n'avait été reconduite que pour un an car l'Ifroa n'était plus en mesure d'exercer sa tutelle en raison de restrictions budgétaires. Les écoles d'art furent obligées de prendre en charge la formation des étudiants dont les diplômes n'étaient plus reconnus par le ministère, faute de convention. Celui-ci résolut la difficulté en confiant à l'Inspection générale de l'enseignement artistique une mission d'évaluation dont s'acquitta en 1989 le service des Musées classés et contrôlés, qui confirma leur agrément, indispensable à la construction du réseau d'ateliers décentralisés. L'Inspection générale avait passé un an plus tôt une convention avec la MST de l'université Paris 1 qui mit simultanément fin à la guerre des formations. Le service de restauration des musées classés ouvrit en 1988 ses ateliers aux étudiants de l'université et mit à la disposition des enseignants les œuvres dont ils avaient besoin pour enseigner la conservation-restauration des peintures sur toile.

Après la décentralisation culturelle, la deuxième série d'événements qui stabilisa le réseau d'enseignement de conservation-restauration dans la décennie suivante fut l'homologation des diplômes des quatre écoles par le ministère de l'Éducation nationale. Toutes les écoles qui avaient ouvert des départements de restauration en province sans passer de convention avec l'Ifroa, comme à Valence ${ }^{65}$ ou Clermont-Ferrand, avaient dû renoncer à leur projet. La discipline se constitua ainsi, par raréfaction de l'offre de formation et diffusion des pratiques contrôlées. Tandis que l'Inspection générale de l'enseignement artistique condamnait les anciens savoir-faire, les spécialistes de la conservation-restauration enseignaient à Paris ou en province ceux qui les remplaçaient. L'homologation des diplômes délivrés par les établissements qui dépendaient du ministère de la Culture par comparaison avec ceux du ministère de l'Enseignement supérieur acheva de réunir les quatre écoles à partir de 1994. Le classement à un niveau de certification des diplômes délivrés par l'Université se faisait automatiquement en fonction des grades et la procédure prévoyait ensuite d'évaluer ceux des autres ministères en comparant leurs référentiels aux leurs, jusqu'à établir un système d'équivalence entre les formations facilitant la promotion sociale ${ }^{66}$. Le fait que le seul diplôme universitaire de conservation-restauration ait été classé au niveau I obligea dans ce contexte toutes les autres filières à revoir leur référentiel pour être homologué par référence au sien. L'Ifroa dut ainsi renoncer au titre de " diplôme d'étude approfondi en restauration d'œuvre d'art $\aleph^{67}$ qui ne correspondait pas à la fin du second cycle universitaire, et les écoles d'art abandonnèrent celui de "technicien supérieur de restauration " pour des raisons inverses. À niveau égal, tous choisirent finalement de mentionner leur spécialisation en "conservation-restauration » qui les rattachait au même paradigme, hormis l'Ifroa qui s'y refusait pour des raisons idéologiques.

Outre la décentralisation et l'homologation, une troisième contrainte administrative explique l'harmonisation de formation à la veille de la loi sur les musées de France. 
L'administration générale du ministère de la Culture estimait à l'époque que la fragmentation et le cloisonnement des services de recherche et de restauration qui dépendaient des différentes directions du patrimoine - archives, bibliothèques, musées, monuments et sites - conduisaient à un gaspillage de moyens qu'un regroupement aurait permis d'éviter. Entre 1990 et 1995, elle lança un audit interne de ses services, appelé «Plan national de Restauration» qui amena leurs responsables à confronter leurs pratiques pour coordonner leurs actions et envisager des rapprochements ${ }^{68}$. L'administration générale tâcha d'unifier leur doctrine durant cette période, comme l'Inspection générale l'avait fait dix ans plus tôt pour les formations. En 1996, cette réflexion aboutit au rattachement de l'Ifroa à l'École nationale du patrimoine où se formaient les conservateurs ${ }^{69}$, puis à la création du Centre de recherche et de restauration des musées de France (C2RMF) en 1998, par fusion du Laboratoire de recherche des musées de France et du Service de restauration des musées de France ${ }^{70}$. Plus de soixante ans après l'adoption officielle du paradigme de la conservation-restauration en 1932, l'administration française suivait ainsi son principe fondateur qui demandait aux conservateurs, aux restaurateurs et aux scientifiques de travailler ensemble. Ce rapprochement imposé par le Plan au sein duquel il existait un groupe de travail sur les formations conduisit enfin l'université de Paris 1 à créer en 1994 un nouveau «diplôme d'études supérieures spécialisées en conservation préventive des biens culturels». Contrairement à la "restauration" et à la conservation "curative", les méthodes holistes de prévention mise au point à l'Iccrom dans les années 1980 portaient moins sur des objets que sur les collections et ne demandaient plus aux spécialistes d'intervenir directement sur elles, mais indirectement en contrôlant les facteurs de dégradation liés à l'environnement ${ }^{71}$.

17 La formation dirigée par D. Guillemard, M. Berducou, C. Laroque, conçue en partenariat avec l'Iccrom et $\mathrm{G}$. de Guichen, s'adressait à tous les professionnels de la conservation et pas seulement aux conservateurs-restaurateurs. Elle eut pour effet de resserrer les liens ténus qui existaient entre tous les experts de la chaîne de transmission patrimoniale ${ }^{72}$ et de renforcer la robustesse du réseau de conservation-restauration dont le développement venait d'être relancé par le Plan national. Mais en dépassant d'anciens clivages, elle en révéla aussi deux autres, latents jusqu'alors. Les conservateurs-restaurateurs attendaient en effet, depuis la création de leurs filières de pouvoir poursuivre leurs études au-delà du second cycle universitaire et la question était pour les responsables de ces formations de savoir comment les orienter. L'université imposait à l'époque de choisir entre un diplôme d'études approfondies, ouvrant sur la recherche et le doctorat, et un diplôme d'études supérieures spécialisées, offrant davantage de débouchés professionnels. En choisissant la seconde voie dans l'intérêt des collections, les responsables de la formation accentuèrent le clivage de la recherche et de la restauration que la création du C2RMF tâchait de résorber et amenèrent les conservateurs-restaurateurs à se diviser en deux groupes en élevant leur niveau de formation. Le concept de prévention leur demandait d'une part de ne plus intervenir directement sur les objets et de renoncer aux méthodes trop interventionnistes qui les liaient encore aux métiers d'art. Mais il élargissait d'autre part leur champ de compétence vers la gestion des collections, le contrôle de l'environnement et l'aménagement des réserves. Il offrait ainsi de nouveaux marchés aux praticiens diplômés de la formation, consistant parfois à planifier les interventions de leurs collègues $^{73}$. Sans constituer une discipline à part entière, la création de ce diplôme fit apparaitre une nouvelle catégorie d'experts, appelés « préventeurs ». 
En 2002, la loi sur les musées de France s'appuya enfin sur ces diplômes pour reconnaître les praticiens autorisés à intervenir sur ses collections ${ }^{74}$. Dès 1932, l'office international des musées avait recommandé de protéger le patrimoine des méfaits de l'ignorance en réglementant l'activité et en protégeant le titre des professionnels ${ }^{75}$. En 1992, le ministère de la Culture déposa au Sénat un premier projet de loi en ce sens, qui fut refusé par le Conseil d'État ${ }^{76}$. Si le ministère a finalement choisi en 2002 de réglementer l'exercice de la profession en s'appuyant sur des diplômes, mais de ne pas protéger le titre des praticiens, c'est en raison de difficultés juridiques liées à l'histoire des institutions autant qu'à des intérêts corporatistes et à des conflits de compétences entre ministères. Les conservateurs-restaurateurs interviennent en effet sur des biens qui dépendent généralement du ministère de la Culture. Mais le statut de profession libérale qu'ils ont majoritairement choisi fait dépendre la protection de leur titre d'un autre ministère ${ }^{77}$. Or il ne peut s'agir du ministère du Commerce et de l'Artisanat, car ces praticiens ne produisent rien, à la différence des maîtres artisans, mais conservent ce qui l'est déjà et prodiguent des conseils dans l'intérêt public. Ils attendent donc que les périmètres des ministères dont dépendent l'exercice de leur activité et la protection de leur titre coïncident et permettent aux autorités de légiférer. Indépendamment de cet acte juridique, la question de leur appellation fait par ailleurs débat sur le plan national, alors qu'elle est tranchée sur le plan international. Les conservateurs du patrimoine leur refusent en effet le nom de " conservateur-restaurateur » proposé en 1984 par le Comité de conservation de l'Icom et s'opposent à leur intégration à la fonction publique. Ils leur proposent de s'appeler des «restaurateurs du patrimoine ", ce que ni eux ni les maîtres artisans des métiers d'art ne peuvent admettre, pour des raisons inverses, mais complémentaires. Les premiers objectent en effet à juste titre que cette expression décrit leur activité et les seconds qu'elle ne correspond pas à la leur, la restauration étant ce que leur discipline sert à éviter. Gageons que ce dernier problème sera réglé dans les prochaines années et que le titre de conservateur-restaurateur sera protégé. Le vœu que firent les pionniers de la discipline dans l'Entre-deux-guerres aura alors été exaucé.

\section{Bilan}

19 Le paradoxe de la restauration dans les métiers d'art liés s'explique ainsi historiquement. Si restaurer n'est pas réparer ou refaire, mais rendre compréhensible la signification d'un objet, les artisans les plus compétents pour le fabriquer ne sont pas les mieux placés pour le restaurer. Ce paradoxe en révèle un autre. Si la France fut parmi les premiers pays à projeter d'ouvrir une école de restauration sous la Première République, on peut s'étonner du fait qu'elle ait été l'un des derniers à le réaliser sous la Cinquième. L'histoire des avanies de ce projet au XIX ${ }^{\mathrm{e}}$ siècle reste à écrire en même temps que celle des métiers d'art. Mais on peut déjà constater que le tournant de celle du $\mathrm{xx}^{\mathrm{e}}$ siècle se situe dans les années 1960, lorsque la création des corps de restaurateurs-spécialistes entra directement en contradiction sur le plan national avec les recommandations de la communauté internationale. Le changement ne s'explique donc pas dans le secteur de la restauration par les revendications des praticiens alors en exercice, qui l'ont plutôt freiné, mais par la création de filières professionnalisantes à l'université et plus tard, par la volonté du président V. Giscard d'Estaing. On peut distinguer quatre périodes dans l'histoire qui suivit cette rupture. La première fut consacrée à la création des filières de conservationrestauration, de 1973 à 1983. La deuxième fut celle de la raréfaction de l'offre de 
formation, entre 1983 et 1988. La troisième fut celle de la coordination des filières, des associations professionnelles et des services de conservation-restauration en France, depuis le lancement du Plan national de restauration en 1990 jusqu'à la création de l'Institut national du Patrimoine en 2001. La dernière commença enfin l'année suivante, avec la loi sur les musées qui s'appuya sur les diplômes de formations pour reconnaître les professionnels autorisés à restaurer ses collections. Entre 1965 et 2002, la France, qui envoyait auparavant les spécialistes de la restauration se former à l'étranger ${ }^{78}$, aura finalement mis moins de trente ans à combler son retard. Si l'on se souvient que les précédents projets de création d'écoles de restauration furent ajournés pour des raisons financières, la question est maintenant de savoir si le pays parviendra à maintenir son offre de formation tandis que les budgets se resserrent et si les professionnels pourront conserver leur code de déontologie dans le paradigme du développement économiquement durable des sociétés ${ }^{79}$.

\section{NOTES}

1. - ICOM. «Le conservateur-restaurateur : une définition de la profession (Copenhague, 1984)». Nouvelles de l'ICOM, 39, 1, 1986, p. 5-6.

2. - APEL, ECCO. Survey of the legal and professional responsibilities of the Conservator-Restorers as regard the other parties involved in the preservation and conservation of cultural heritage = Étude des responsabilités légales et professionnelles des conservateurs-restaurateurs au regard des autres acteurs de la sauvegarde et de la conservation du patrimoine culturel. Rome : Apel, 2001.

3. - LEVEAU, Pierre. «L'enquête sur la formation des restaurateurs dans l'Entre-deux-guerres : transformation d'un métier et reconnaissance d'une profession (1929-2011)». CeROArt, 9, 2014 : http://ceroart.revues.org/3772 [consulté 16/07/14].

4. - MAURER, Robert. "La défense du patrimoine artistique et la formation des restaurateurs ». Mouseion, 20, IV, 1932, p. 142-146.

5. - WEBER, Max. Essai sur la théorie de la science. Paris : Plon, 1965.

6. - Journal officiel de la République française (JORF) $n^{\circ} 299$ du 27 décembre 2003, p. 22260, texte $\mathrm{n}^{\circ}$ 34. Arrêté du 12 décembre 2003 fixant la liste des métiers de l'artisanat d'art. NOR : ECOA0320025A.

7. - Bulletin officiel, Éducation nationale $\mathrm{n}^{\circ} 134$, novembre-décembre 2002, p. $38-40$ : Circulaire $\mathrm{n}^{\circ}$ 2002/021 du 24 décembre 2002 relative à la restauration des biens des collections des musées de France.

8. - JORF $n^{\circ} 79$ du 3 avril 1998, p. 5172. Décret $n^{\circ} 98-247$ du 2 avril 1998 relatif à la qualification artisanale et au répertoire des métiers, NOR : ECOA9720014D.

9. - JORF $\mathrm{n}^{\circ} 100 \mathrm{du} 28$ avril 2002, p. 7742, texte $\mathrm{n}^{\circ} 45$. Décret $\mathrm{n}^{\circ} 2002-628 \mathrm{du} 25$ avril 2002 pris pour l'application de la loi $\mathrm{n}^{\circ} 2002-5$ du 4 janvier 2002 relative aux musées de France, titre II, ch. 3 , art. 13, NOR : MCCX0200050D.

10. - Voir le site : http://www.unma.fr/ [consulté le 13/05/2016].

11. - Voir le site : http://www.ffcr.fr/ [consulté le 13/05/2016].

12. - ECCO. Professional Guidelines (II) : Code of Ethics [document électronique], 2002: http:// www.ecco-eu.org/about-e.c.c.o./professional-guidelines.html. [consulté 15/07/14]. 
13. - DAVALLON, Jean. Le don du patrimoine : une approche communicationnelle de la patrimonialisation . Paris : Hermès science publications-Lavoisier (Communication, médiation et construits sociaux), 2006.

14. - Arch. nat., Bureau de la diffusion des métiers d'art, 19900054, art. 6 ; 19930369, art. 12-15.

15. - Arch. nat., Société d'encouragement à l'art et l'industrie, 38 AS 1-44, 1889-1974.

16. - UNESCO. Cinquième session du Comité intergouvernemental de sauvegarde du patrimoine culturel immatériel, Nairobi, Kenya, 15-19 novembre 2010.

17. - BOILEAU, Étienne. Les métiers et corporations de la Ville de Paris : XIII ${ }^{e}$ siècle, le Livre des métiers d'Étienne Boileau. Éd. René de Lespinasse et François Bonnardot. Paris : Imprimerie nationale (Histoire générale de Paris), 1879.

18. - ICOM. Op.cit., art. 4.1.

19. - UNESCO. L'action normative de l'UNESCO, Vol. II : Conventions, recommandations, déclarations et chartes adoptées par l'Unesco (1948-2006). Leyde-Boston/Paris : Martinus Nijhoff/Unesco, 2007.

20. - LEVEAU, Pierre. "Le souvenir de la Grande Guerre dans les réseaux de conservation de l'Entre-Deux-Guerres. Une préhistoire du Bouclier bleu ", In Situ [En ligne], 23 | 2014, mis en ligne le 21 février 2014, consulté le 13 mai 2016. URL : http://insitu.revues.org/10903 ; DOI : 10.4000/ insitu.10903.

21. - JORF $n^{\circ} 0179$ du 3 août 2005, p. 12639, texte $n^{\circ} 2$, art. 23. Loi $n^{\circ} 2005-882$ du 2 août 2005 en faveur des petites et moyennes entreprises, NOR : PMEX0500079L.

22. - LEVEAU, Pierre. "L'évolution du concept de restauration au $\mathrm{XIX}^{\mathrm{e}}$ et $\mathrm{XX}^{\mathrm{e}}$ siècles ». Conservation-restauration des biens culturels : revue de l'Araafu, 25, 2007, p. 3-11.

23. - NF EN 15898, Conservation des biens culturels - Principaux termes généraux et définitions correspondantes, Df 3.3.7. Paris : Afnor, décembre 2011.

24. - MOHEN, Jean-Pierre. Les sciences du patrimoine: identifier, conserver, restaurer. Paris : Odile Jacob, 1999.

25. - [OFFICE INTERNATIONAL DES MUSÉES]. «L'activité de l'Office international des musées : résolution adoptée par la Commission internationale de coopération intellectuelle le 23 juillet 1932 ». Mouseion, 21/22, 1933, p. 274-276.

26. - ÉMILE-MÂLE, Gilberte. Pour une histoire de la restauration des peintures en France. Paris : éd. Somogy-INP, 2009.

27. - Archives des musées nationaux (AMN) (transférées en 2015 aux Archives nationales, Pierrefitte), Projet non réalisé de création au Louvre d'une école de restauration de tableaux, p. 2, 1936.

28. - [OFFICE INTERNATIONAL DES MUSÉES, et al.]. « Pour une éducation professionnelle des restaurateurs d'œuvres d'art ». Mouseion, 19, III, 1932, p. 83-85.

29. - BRUCY, Guy. Histoire des diplômes de l'enseignement technique et professionnel (1880-1965). Paris : Belin, 1998.

30. - AMN, Concours de recrutement des restaurateurs, O5E, 1935.

31. - AMN, Concours de recrutement des restaurateurs, P.16, 1935.

32. - BAZIN, Germain. «Projet sur la création d'une école de restauration, 1942 ». Dans BOTLAN, Magalie. Germain Bazin et la restauration des peintures. Mémoire de l'École du Louvre, 2001-2002, Annexe 4.

33. - KAPLAN, Steven, MINARD, Philippe (éd.). La France, malade du corporatisme? Paris : Belin, 2004.

34. - JORF du 2 août 1961, p. 7147-7148. Décret $n^{\circ}$ 61-838 du 28 juillet 1961 relatif aux dispositions statutaires communes applicables aux ouvriers professionnels des administrations centrales des ministères et administrations assimilées.

35. - BERGEON, Ségolène. «Du métier de restaurateur à la discipline de la restauration ». Dans CENTRO EUROPEO DI RICERCA SULLA CONSERVAZIONE E SUL RESTAURO DI SIENA (CERR). Carte, 
risoluzioni e documenti per la conservazione ed il restauro: Siena, 14-15 marzo 2003. Pisa Ospedaletto: Pacini, (Quaderni del Cerr, 3), 2006, p. 163-171.

36. - Arch. nat., Direction des Musées de France, 19920481, Art. 38 et 43.

37. - JORF, décret $n^{\circ} 64-269$ du 20 mars $1964 ; n^{\circ} 64-1012$ du 24 septembre $1964 ; n^{\circ} 6$ 5-855 du 24 septembre $1965 ; n^{\circ} 66-546$ du 22 juillet 1966.

38. - JORF du 13 juin 1969. Règlement des concours de recrutement dans le corps de la restauration d'art des musées de France.

39. - COREMANS, Paul (dir.). L'Agneau mystique au laboratoire: examen et traitement. Anvers: De Sikkel, 1953, p. 10.

40. - ICOMOS. Charte internationale sur la conservation et la restauration des monuments et des sites. Venise, $\mathrm{II}^{\mathrm{e}}$ congrès international des architectes et des techniciens des monuments historiques. Venise, 1964, art. 9-13.

41. - PLENDERLEITH, Harold J. «A history of conservation ». Studies in Conservation, 43, $\mathrm{n}^{\circ} 3,1998$, p. 129-143.

42. - THOMSON, Garry (dir.). Recent Advances in Conservation. Contributions to the IIC Rome Conference, 1961. Londres : Butterworths, 1963, p. 197-213.

43. - Archives de l'ICOM, 1965, Maison de l'Unesco, Paris.

44. - JORF du 13 novembre 1968. Loi $n{ }^{\circ} 68-978$ du 12 novembre 1968 d'orientation de l'Enseignement supérieur.

45. - Arch. nat. (Fontainebleau), Culture, 19790477, Art. 19-20.

46. - AMN, Germain Bazin, O* 30-464.

47. - JORF du 16 juillet 1971, Annexe C1, PL. 170-171 pour l'éducation, et Annexe C3, PL. 182 pour le développement culturel.

48. - JORF du 20 février 1971, p. 1752. 13 janvier 1971. Arrêté portant création d'une maîtrise de sciences et techniques.

49. - MINISTÈRE DE L'ÉDUCATION NATIONALE. Groupe d'étude des formations supérieures - 14. Paris : La Documentation Française, 1972.

50. - JORF du 29 octobre 1977, p. 7148. 14 octobre 1977. Déclaration à la Préfecture de Police. Institut français de restauration des œuvres d'art.

51. - Arch. nat., Délégation aux Arts plastiques, 19980199, art. 1, 16 et 23.

52. - CRESSON, Édith. Étude sur la création d'un institut de restauration. Paris : secrétariat d'État à la Culture, service des Études et recherches, direction des Musées de France, novembre 1974.

53. - DEHAYE, Pierre. Rapport au Président de la République sur les difficultés des métiers d'art (IV, C, 2). Paris : La Documentation française, 1976.

54. - Mission des archives du ministère de la Culture (MAMC). Centre de recherche et de restauration des musées de France, 09 V 26, art. 9-11.

55. - AMN, Commission pour la conservation du patrimoine, 7 KK 1.

56. - COURAL, Jean. " Note sur l'histoire de la restauration ». Dans CIESLEWICZ, Roman, MUSÉE DES ARTS DÉCORATIFS. Les métiers de l'art: formation, tradition, restauration, création. Cat. expo. Paris, musée des Arts décoratifs, novembre 1981-mars 1981. Paris : musée des Arts décoratifs, 1980, p. 20-22.

57. - Arch. nat., Politique pour le développement de la formation aux métiers d'art (1975-1997), 20020469, art. 1-15.

58. - JORF du 19 janvier 1978. Arrêté portant création d'un diplôme national supérieur d'expression plastique.

59. - Arch. nat., Inspection générale de l'enseignement artistique, 19860305/30.

60. - BAZIN, Germain, ÉMILE-MALE, Gilberte, BERGEON, Ségolène. Comprendre, sauver, restaurer. La campagne de restauration des primitifs italiens du Musée du Petit Palais 1966-1976. Avignon : musée du Petit Palais, 1976 ; FAILLANT-DUMAS, Lola. Au-delà du visible : 10 tableaux Campana aux rayons X. Exposition du LRMF, avril-octobre 1980. Avignon : musée du Petit Palais, 1980. 
61. - LEVEAU, Pierre. «Problème de l'apolitique de la conservation-restauration ». Conservationrestauration des biens culturels : revue de l'Araafu, 29, 2011, p. 5-26.

62. - RIZZARDO, René. La décentralisation culturelle. Rapport au ministre de la Culture et de la Communication. Paris : La Documentation française, 1990.

63. - Arch. nat., Inspection générale de l'Enseignement artistique, 19860305/51.

64. - MAMC. Centre de recherche et de restauration des musées de France, 09 V 26, Art. 1-3 et 23-24.

65. - BAYLE, Gérard. Peindre... Conserver. Exposition $1^{\text {er }}$ mars-18 avril 1982. Valence : Atelier de restauration de l'école d'art et musée de Valence, 1982.

66. - VENEAU, Patrick, MAILLARD, Dominique. " "Les niveaux de formation" à l'heure européenne : un examen à partir de l'homologation des titres ». Formation Emploi : Revue française de sciences sociales, 102, avril-juin 2008, p. 71-84: http://formationemploi.revues.org/2286 [consulté le 13/05/2016].

67. - JORF du 7 mai 1988, p. 6467.

68. - MAMC. Centre de recherche et de restauration des musées de France, 08 V 151, art. 31-32.

69. - Arch. nat., Institut français de restauration des œuvres d'art, 20040011, art. 57-64; 20040052, art. 190-192.

70. - MAMC. Centre de recherche et de restauration des musées de France, 08 V 95, art. 1-7, 12-14 et 28 .

71. - GUILLEMARD, Denis (dir.). La conservation préventive: colloque sur la conservation-restauration des biens culturels, Paris, 8-10 octobre 1992. Paris : Araafu, 1992.

72. - GUICHEN, Gaël de. « Preventive conservation: a mere fad or far-reaching change? ». Museum International, 51, 1999, p. 4-6: http://onlinelibrary.wiley.com/doi/10.1111/1468-0033.00183/ abstract [consulté le 13/05/2016].

73. - GUILLEMARD, Denis (dir.). « Nouveaux métiers du patrimoine et conservation préventive ». Conservation-restauration des biens culturels, Cahier technique, $\mathrm{n}^{\circ} 6,2000$.

74. - JORF du 28 avril 2002, p. 7744. Article 13 du décret d'application de la loi sur les musées de France.

75. - GRAUL, Richard. " Pour une éducation professionnelle des restaurateurs d'œuvres d'art ». Mouseion, 21-22, 1933, p. 269-270.

76. - SÉNAT. «Projet de loi $\mathrm{n}^{\circ} 202$ relatif aux musées, aux établissements publics territoriaux à vocation culturelle et aux restaurateurs du patrimoine ", rattaché au procès-verbal de la séance du 23 décembre 1992. Enregistré à la Présidence du Sénat le 21 janvier 1993.

77. - Journal officiel de l'Union européenne du 30 septembre 2005, p. 22. Directive européenne $n^{\circ}$ 2005/36/CE relative à la reconnaissance des qualifications professionnelles, NOR : 305L0036.

78. - VOLLE, Nathalie. «Cesare Brandi et Germain Bazin : restauration et modernité ». Dans BASILE, Giuseppe (éd.). Cesare Brandi, la restauration: méthode et études de cas. Paris: INP/éd. Stratis, 2007, p. 277-309.

79. - FAIRCLOUGH, Graham. «Les nouvelles frontières du patrimoine ». Dans Le patrimoine et audelà. Strasbourg: Éd. Conseil de l'Europe, 2009, p. 31-45: http://www.coe.int/t/dg4/ cultureheritage/heritage/identities/PatrimoineBD_fr.pdf [consulté le 13/05/16]. 


\section{RÉSUMÉS}

Qu'est-ce qui distingue les maîtres-artisans des métiers d'art spécialisés dans la restauration du patrimoine et les professionnels de la conservation-restauration des biens culturels ? Il paraît indispensable de revenir sur l'histoire de quatre formations pour comprendre comment la conservation-restauration des biens culturels s'est professionnalisée en France en rompant avec la tradition. Il s'avère que ce tournant s'explique moins, dans ce secteur particulier, par les revendications professionnelles des corps de restaurateurs spécialistes déjà constitués dans différentes institutions patrimoniales, que par la volonté du ministère de la culture de réintroduire la France dans les réseaux internationaux de conservation créés sous l'égide de l'UNESCO.

What is the difference between master craftsmen specialised in the restoration of heritage artefacts and the professionals of the conservation-restoration of cultural properties? To answer this question, it is necessary to reconsider four training programmes, in order to understand how the conservation-restoration of cultural heritage has become a profession in France by breaking with tradition. In this particular sector, it appears in fact that this historic turning point is to be explained not so much by the professional claims of existing restorers' organisations in various heritage institutions, as by the Ministry of Culture's political will to reintroduce France into the international networks of conservation created under the aegis of UNESCO.

\section{INDEX}

Keywords : conservation-restoration, craft professions, profession, training, history

Mots-clés : conservation-restauration, métiers d'art, profession, formation, histoire

\section{AUTEUR}

\section{PIERRE LEVEAU}

Membre associé au Centre d'épistémologie et d'ergologie comparatives (CEPERC), UMR 7304

leveau.p@wanadoo.fr 